\title{
Blockchain Technology and Cryptocurrencies: Opportunities for Postal Financial Services
}

Christian Jaag

Christian Bach

Swiss Economics Working Paper 0056

August 2016

Swiss Economics SE AG

Abeggweg 15

CH-8057 Zürich

T: +41 (0)44 5005620

F: +41 (0)44 5005621

office@swiss-economics.ch

www.swiss-economics.ch 


\section{Introduction}

This paper explores opportunities arising from blockchain technology for postal operators (POs). ${ }^{1}$ Blockchain technology has lately received a lot of interest by the media and the industry, especially in financial services. ${ }^{2}$ In the past years an entire ecosystem of new companies has developed, offering hundreds of different blockchain applications. Blockchains are a new kind of decentralized, secure and fast means of record-keeping. The first application of blockchain technology are cryptocurrencies like Bitcoin, which have become an alternative to commodity money and fiat money, but POs may be able to exploit this technology in a number of different ways.

Contrary to traditional currencies, cryptocurrencies neither have physical form nor are they guaranteed or backed by any central authority. They are created by their users and attain value by usage and the confidence of those participating in the respective system. Cryptocurrencies are associated with their own payment systems, which allows for payments between individuals digitally without relying on central institutions, intermediaries or further infrastructure as required for conventional payment systems. Besides Bitcoin, there are over 200 further cryptocurrencies that are essentially copying the Bitcoin protocol with some minor changes or improvements. While their legitimacy as currencies has been questioned due to their high exchange rate volatility, the significant potential of blockchain technology for applications far beyond payments is undeniable.

As POs typically have a role as financial intermediaries and act in an international and increasingly digital environment, decentralized blockchain-based payment-systems potentially may be of particular interest to them. In fact, as the post has a wide network of access points and is highly trusted by the general public, it may be well-suited to offer services which counter some disadvantages of decentralized payment systems and cryptocurrencies, while retaining the benefits of their technology. By turning to these new technologies, POs may extend their role as a financial intermediary with new domestic and international services. Furthermore, POs may even issue their own cryptocurrency to protect customers from the high exchange-rate volatility of current cryptocurrencies.

The paper proceeds as follows. Section 2 presents a brief characterization of decentralized payment-systems and cryptocurrencies. Also, an overview of government regulation for cryptocurrencies is provided with focus on the United States. Furthermore, several opportunities and challenges for individuals and companies related to cryptocurrencies are presented. In Section 3 it is argued that POs could benefit from including financial blockchain applications in their business model. In Section 4 the idea of Postcoin, a novel concept for a postal cryptocurrency, is presented. Section 5 briefly discusses non-financial applications of blockchain technology for POs. Finally, a conclusion is offered in Section 6.

\footnotetext{
${ }^{1}$ National incumbents and their competitors.

2 See e.g. The Economist (2015). Also POs have become interested in the technology, see e.g. USPS (2016).
} 


\section{Blockchain technology and cryptocurrencies}

Blockchain technology was originally created as a way to transfer value, specifically within the context of the digital currency Bitcoin. As such, uses of blockchain technology include payments and other financial transactions. ${ }^{3}$ Blockchains enable peer-to-peer transactions by removing the need for a trusted intermediary to verify the transactions, and to delay the transaction while it is being verified. Hence, there is no bank or other single third party keeping the ledger and verifying the transaction - no one entity controls the ledger. Instead, the network, as a whole, verifies the transactions through a decentralized consensus mechanism.

A blockchain makes this possible by being a decentralized ledger. This public ledger is not so different from the ledger that traditional financial institutions maintain, with a record of who owns what. Blockchain technology combines two ideas: First, defining a digital token (i.e. a coin of a corresponding currency) as a chain of transactions makes it possible to solve the problem of double spending, because ownership is defined as having received a coin in the past and being its latest recipient. In contrast, simple possession of a code would not be suitable, because each code can be duplicated.

Second, by using a validation mechanism like proof of work ("mining"), there is only one accepted transaction history. There are two types of participants in a blockchain network: Nodes and miners. Nodes verify the legitimacy of blocks and keep a copy of the blockchain. They also create transaction records (e.g. transferred quantity of currency, addresses involved, proof that the transaction is valid, time of transaction), verify other nodes' transactions and spread them over the network. Mining is the process of adding transactions to the blockchain. Miners are specialized nodes that pick up transaction records, verify them and generate new blocks by performing cryptographic functions. In the case of Bitcoin, miners find a block every ten minutes on average and get awarded with new bitcoins in return. ${ }^{4}$ Once a transaction has been added to a block and the block is chained to the previous block, it is settled irrevocably. ${ }^{5}$ As all users who operate a node of the network must agree on every legitimate transaction ${ }^{6}$ in the past (by

${ }^{3}$ Further applications will be outlined in Section 5.

${ }^{4}$ In Bitcoin, there are two kinds of incentives for miners: The first is new bitcoins. The reward started at 50 bitcoins per block; this value halves every 210,000 blocks, such that the total supply of bitcoins asymptotically approaches 21 million. Users can also attach fees to their transactions (the fee typically amounts to the equivalent of a few USD cents). Miners use the fees to decide which transactions to include in a block and collect the fees (see Jaag and Haller, 2016). Currently, the miners' revenue from fees is much lower than the block reward. Since there is a maximum block size, block capacity becomes scarcer with increased usage. As a result, transaction fees become a more important source of revenue for miners (unless the maximum block size is increased which would require a change in the consensus protocol).

${ }^{5}$ The settlement time of ten minutes on average is much faster than with any non-cash financial transaction which may take days or - in the case of credit cards - even weeks.

${ }^{6}$ By participating in the network, nodes know of all transactions and blocks. Therefore, operators of nodes do not rely on third parties for checking whether a payment to them is legitimate and can be trusted. This is the incentive to run a node. 
passing them on to other nodes), determining who owns a specific currency unit is uncontroversial, and without the need to trust anybody. Essentially, a decentralized register determines the number of currency units belonging to the person who is able to prove that he is entitled to spend them.

Generally, blockchains fall into two groups: public and private. In a public blockchain like Bitcoin's, the right to alter the ledger by participating in the consensus mechanism is open to anyone. Transactions are publicly available for anyone to read. Alternatively, in a consortium or private blockchain, the right to alter the ledger by participating in the consensus mechanism is restricted to pre-selected individuals or institutions. Transactions may be either publicly available or restricted to a select number of participants.

\subsection{Characteristics}

Decentralized payment-systems enable peer-to-peer transactions, i.e. two individuals can exchange value without relying on a centralized third party. This is a major difference to existing payment solutions, as no financial intermediary is required for a transaction. In comparison, for a bank transfer two banks need to exchange money on behalf of their customers. Moreover, allowing an institution to transfer money, the transacting parties not only give away private information related to the transaction, but also give it access to their funds as well as to personal data. ${ }^{7}$ Using a decentralized payment-system - without any intermediary - transaction costs are low, while no access to funds or personal information is given away to any third party. ${ }^{8}$

An important implication, which follows from a decentralized ledger, is the irreversibility of transactions. Once a payment is issued, it cannot be reversed. The only way to recover the claim is by asking the receiver to pay back the same amount in a new transaction. The irreversibility follows from the fact that each transaction is added to the blockchain by miners, which in turn cannot be altered but only extended. Therefore, payment in cryptocurrencies is similar to a cash payment, but does not have to be conducted over-the-counter. However, in contrast to the existing non-cash payment systems such as credit card or bank transfer (which are all reversible), the risk of transaction is shifted from receiver to sender via this irreversibility. ${ }^{9}$

Another distinguishing property of decentralized payment-systems is the pseudo anonymity of the transacting parties. When transferring cryptocurrencies, there is no need to disclose any personal information to the public or any third party whatsoever. This substantially reduces the risk of identity theft and fraud common with other forms of payment such as credit cards. Users can act under one or several pseudonyms without any obvious links to their true person. The pseudo anonymity of cryptocurrencies has

\footnotetext{
${ }^{7}$ Especially with credit card payments, this creates the risk of theft.

8 The success of blockchain-based transactions systems suggests that the cost of processing a transaction is less than the cost per transaction of the equivalent effort by the banking sector and, if need be, monetary authorities, or that additional costs are outweighed by the benefits in speed, security, and privacy.

${ }^{9}$ Cf. Jaag and Bach (2015) for a microeconomic analysis of the irreversibility characteristics of cryptocurrencies.
} 
given rise to some discussion about illegal usage. ${ }^{10}$ This is a valid concern and thus an important topic for regulation.

Moreover, decentralized payment-systems are not bound by any geographical limit: Because of the virtual nature of the payment system, it does not matter whether an individual sends cryptocurrencies to a neighbor or to someone on the other side of the world. In contrast, it is often difficult to use traditional payment systems to transact across borders, since the financial intermediaries are bound by country-specific regulation and differing exchange rates. Decentralized payment-systems transcend state borders: essentially, they form a global payment system instead of several national ones.

Public blockchain-based payment systems need to provide incentives to their users to assist in securing the system. Consequently, it is not possible to use such systems for payments without using some currency as possible reward for securing the network. This can be accomplished by issuing units of a respective cryptocurrency, which can then by "mined" as a "block reward" by those who prove that they contribute to securing the network. ${ }^{11}$

A key property of cryptocurrencies, which significantly distinguishes it from traditional currencies, is the lack of a central money issuer. Cryptocurrencies are created in a decentralized process without any authority controlling the distribution of new units. Hence, there also exists no institution that could actively conduct monetary policy in the system. Consequently, cryptocurrencies are also not backed by any assets that central banks in a fiat or gold-backed system keep on their balance sheet. Exchangeability separates cryptocurrencies from other virtual concepts like frequent flyer miles or Facebook credits, which are neither freely tradable between people nor exchangeable against services outside the issuing company.

\subsection{Regulation}

Cryptocurrencies their payment systems are hard for governments to regulate. First, there is no central point of access. Second, decentralized payment-systems allow for international money transmission without any concern for national borders. Therefore, regulation would need to be coordinated across countries.

Decentralized payment-systems do not exhibit any central point of access for governmental interference or law enforcement. For instance, it is difficult for authorities to seize money holdings in cryptocurrencies since the entire system is pseudo anonymous. While a government may freeze bank accounts, it cannot do so with decentralized payment-systems. However, institutions and companies offering services related to decentralized payment-systems can be subjected to regulation, as they provide central access points. For instance, currency exchanges, which present a gateway between traditional currencies and cryptocurrencies, can be forced to abide by government regulation such

${ }^{10}$ A specific concern is ransomware that disables the computer unless a ransom is paid. Ransomware cannot accept PayPal or credit cards since those transactions are traceable. The increasing adoption of cryptocurrencies makes using them worthwhile.

${ }^{11}$ See the introduction to Section 2 above. 
as anti-money-laundering law. Law enforcement against Mt.Gox, a former exchange platform, is an example for this.

In the United States, the Financial Crimes Enforcement Network (FinCEN) has issued a first guidance relating to the regulation of money service businesses in March 2013 (see FinCEN, 2013). Different rules apply to users and exchanges. Individuals who use cryptocurrencies to purchase or sell goods, do not fall under FinCEN's regulation. In contrast, cryptocurrency exchanges are considered to be money service businesses and therefore need to comply with FinCEN regulation. This regulation entails anti-moneylaundering law. Subsequent to this first guidance, FinCEN (2014a, 2014b) has provided additional clarification for services related to cryptocurrencies: miners and software providers do not fall under its regulation.

The New York State Department of Financial Services issued a so-called BitLicense, i.e. a business license of cryptocurrency activities. After it came into effect in August 2015, several Bitcoin companies announced they were stopping business in New York State because of the new regulations. In September 2015, the first BitLicense was issued to Circle Internet Financial (see NYDFS, 2015).

\subsection{Opportunities}

Cryptocurrencies, such as Bitcoin, have innovative features either as a currency, payment system or, more generally, as a technology. With their unique characteristics, they bear the potential to substantially influence the existing financial system. Several opportunities could unfold for individuals, businesses and the economy as a whole.

By substituting traditional payment methods (which rely on financial intermediaries) by cryptocurrencies, it is possible to decrease transaction fees and therefore reduce the costs from non-cash payments. This especially applies to online businesses. Furthermore, decentralized payment-systems provide a quick low-cost way for sending money directly from person to person. For example, with traditional remittance services, emigrant workers pay an average of 12 percent in fees to transfer money back to relatives in Sub-Saharan Africa (see Watkins and Quattri, 2014).

The combination of low transaction costs with fast, easy usage can provide new methods of revenue schemes based on microtransactions. For instance, with cryptocurrencies it becomes possible to add a tipping system to online services such as blogs or to crowdfunded projects. Previously, small transactions have not been worthwhile, as the transaction costs outweighed the benefits or even the value of the transactions itself.

For individuals and companies it can be advantageous that transactions with cryptocurrencies are irreversible. For instance, payments by credit cards can be reversed after the purchase. Online merchants are thus exposed to the risk that customers reverse their payments after the respective order has already been shipped. In fact, payment irreversibility may strengthen e-commerce by reducing its overall risk, if merchants have more reputation to lose than customers (see Jaag and Bach, 2015). In 2016, there are more than 100,000 merchants accepting Bitcoin, including Microsoft, Overstock, Newegg, DISH and Expedia (Nasdaq, 2016). 
Cryptocurrencies may also offer an alternative store of value for countries with unstable currencies. ${ }^{12}$ For instance, in high-inflation countries, it may be beneficial to hold cryptocurrencies as assets in addition to national currency. Moreover, cryptocurrencies do not fall under the authority of government, and can thus not be devaluated or held back for fiscal or other purposes. A recent example is Argentina where the government devaluated the domestic currency to counter the country's trade deficit (see Coindesk, 2014).

\subsection{Challenges}

In spite of several opportunities and a substantial innovation potential, quite some challenges associated with the nature and the use of cryptocurrencies and decentralized payment-systems remain. They open up new opportunities for business cases by trusted institutions like POs.

One major challenge is that it is hard to find out whether cryptocurrencies are a serious technology or just a large scam. Hence, potential users might want to understand decentralized payment-systems before starting to use them. Even though most of them do not understand the traditional financial system either (beyond an intuition and reasonably favorable experience), individuals and merchants who do not comprehend the mechanics of the underlying technology may hesitate to enter the cryptocurrency system due to a lack of trust in the system. Ignorance about decentralized payment-systems is still rather widespread. For instance, according to a survey by The Street (2014), three fourth of the questioned persons in the United States indicated that they are not familiar with Bitcoin at all.

Regulatory uncertainty also severely restricts widespread adoption of cryptocurrencies. Indeed, lack of clear governmental guidance represents one of the main challenges for cryptocurrencies at the moment. Generally, regulation in the financial sector usually demands high compliance and risk management efforts from financial intermediaries. Regulatory uncertainty is thus especially problematic because handling cryptocurrencies may result in the involuntary provision of financial services, subject to government regulation of financial intermediaries. New businesses acting in the uncharted territory of cryptocurrencies are hence exposed to the risk of being prosecuted.

Security concerns about handling and storing cryptocurrencies are another major challenge for users. Adversaries may gain access to their wallet and steal their money. Currency exchanges and companies that store cryptocurrency for their customers are vulnerable, too. If such companies are attacked, users may lose all money on their account. The most prominent example of cryptocurrency theft happened to the exchange platform Mt. Gox, which lost the equivalent of approximately USD 365 million in Bitcoin (see Bloomberg, 2014b). Following this incident, Mt. Gox declared bankruptcy and it is likely that users will not be able to reclaim their assets.

Limited availability of cryptocurrency as well as the lack of trusted exchanges pose further problems for adoption. Currently, access to cryptocurrencies is provided only by online exchanges or personal trade. Exchange platforms are mostly new start-up firms

${ }^{12}$ Exchange rates of currencies used in industrialized countries are usually more stable than cryptocurrencies. However, in developing countries, local currencies may be more volatile. 
with little reputation and no representation in the real world. However, people not familiar with online services would prefer to exchange currencies over the counter or use credit or debit cards. The introduction of cryptocurrency teller machines provides a partial solution, but it will take some time for their reach to be at a satisfactory level.

Finally, high exchange rate volatility also poses an issue for cryptocurrencies. For instance, the Bitcoin exchange rate against the USD plunged about 35 percent in December 2012 after rumors had come up that Chinese regulators were to ban cryptocurrencies in their country (see Bloomberg, 2014a). Users and merchants may not be willing to bear price fluctuation risks, which could adversely affect the value of sales. However, they do not necessarily need to bear the exchange rate fluctuation of cryptocurrencies. They can protect themselves from such risk by using hedge funds or service providers that convert cryptocurrencies into traditional currencies instantly. For instance, merchants who accept cryptocurrencies from their customers can immediately exchange them to a traditional currency. Thereby, these merchants benefit from decentralized payment-systems without being exposed to the volatility of corresponding cryptocurrencies.

As more companies and customers adopt them and their liquidity increases, cryptocurrencies are likely to gain in stability as a currency. Besides, a substantial part of the current fluctuations are due to regulatory and market uncertainties - both of which will decrease as regulatory guidance becomes clearer.

The challenges of cryptocurrencies can be summarized as follows. A lack of trusted and established institutions in the realm of decentralized payment-systems unleashes a feeling of risk and necessitates a high degree of personal responsibility, which users are not used to in conventional payment systems. These key challenges could be tackled by trusted firms, which offer cryptocurrency related assistance and services.

Table 1 provides an overview of the discussed opportunities and challenges of cryptocurrencies and decentralized payment-systems.

\begin{tabular}{|c|c|}
\hline Opportunities & Challenges \\
\hline - $\quad$ Easy-to-use & - $\quad$ Limited Availability \\
\hline - Privacy & - Security in usage \\
\hline - Low transaction fees & - Reliable institutions \\
\hline - Microtransactions & - Regulatory uncertainty \\
\hline - Irreversibility & - Limited prevalence \\
\hline - Alternative store of value & - Exchange rate volatility \\
\hline
\end{tabular}

Table 1: Opportunities and challenges of cryptocurrencies and decentralized payment-systems

\section{Potential applications for POs}

Blockchains and cryptocurrencies provide new technologies which POs can use to better provide financial and non-financial services. Moreover, the challenges associated with the use of these technologies for individual users open up opportunities for new intermediaries like POs as trusted institutions. 
Many POs face legal universal service obligations, requiring them - among other things - to provide access to post offices within reasonable distance. In 2013, with 663,210 post offices globally, the network of postal outlets was the densest retail network in the world (see Universal Postal Union, 2014). There currently exist two major challenges for traditional POs: Indirect competition from electronic substitutes in the letter segment and direct competition in the parcel segment.

The first challenge results from the increasing use of email and other Internet based services for communication purposes. Since the end of the 1990s, physical mail volumes have declined in most industrialized countries. Consequently, posts face the challenge of operating dense networks of branches, being able to serve the population in proximity, while suffering from a decreasing letter demand.

The second challenge concerns the continuously growing sector of e-commerce. From the perspective of POs, e-commerce constitutes a unique opportunity and a rare growth area. Indeed, the global domestic postal parcel traffic has doubled during the last 20 years. However, liberalization of the parcel segment has attracted considerable competition.

There seems to be a well-suited match between cryptocurrencies and posts in view of the above-mentioned challenges (see Jaag and Bach, 2013). On the one hand, cryptocurrencies and decentralized payment-systems are still lacking a physical interface that is easily as well as generally accessible to the public, while on the other hand POs dispose of nation-wide networks of branches with declining usage in their traditional core business. A natural opportunity for Posts seems to flow from using their network as a bridge between traditional currencies and the virtual world of cryptocurrencies by offering local exchange and transaction services. Also, the technology of decentralized paymentsystems could be used to innovate and advance existing financial services. Some domestic and international opportunities for POs that might ensue from an implementation of cryptocurrencies into their business model are now presented.

\subsection{Domestic opportunities}

Cryptocurrencies and decentralized payment-systems could extend the financial role of POs by enabling the provision of new services in their respective home markets.

\subsubsection{Retail solutions and e-commerce}

Cryptocurrencies appear to fit well the global rise of e-commerce. In 2012, sales in ecommerce already topped USD 1 trillion and are expected to rise annually by approximately 15 percent in the next few years (see eMarketer, 2013). On the one hand, this means that electronic payment methods will further gain in importance, as there is a physical separation between merchant and customer. On the other hand, this separation also increases the demand for postal logistic services, as the purchased goods need to be delivered to customers. Since cryptocurrencies, especially Bitcoin, are increasingly used in e-commerce, service provision with regards to cryptocurrencies could attract new customers to the post offices for parcels. In particular, persons owning neither a credit card nor a bank account would thus be enabled to access e-commerce via exchanging traditional currencies for cryptocurrencies. As a single intermediary between merchants and customers providing parcel delivery but also facilitating the financial transaction in e-commerce, POs would be able to reduce coordination needs and to offer more efficient 
e-commerce solutions. By combining their traditional strength in physical delivery with easy and low-cost payment services, POs may facilitate e-commerce and actually contribute to its further growth.

\subsubsection{Services for individuals}

POs could also aim towards becoming a leading service point for remittances in cryptocurrencies and corresponding monetary transfers through decentralized paymentsystems. Although it is possible for individuals to send, for example, Bitcoin without intermediary, there is still a role for the post as money transmitter. Combined with a postal account, POs could provide an interface for their clients to easily send money without having to understand decentralized payment-systems in detail.

The adoption of additional financial services also seems to correlate with the growth strategies of the financial branch in the postal sector. Indeed, POs already have considerable financial knowledge, which could be transferred to offer new financial services. Together with their extensive physical network, they are in strong position to not only serve domestic but also international markets.

\subsection{International opportunities}

As decentralized payment-systems are not limited by national borders, they could provide the infrastructure for commercial and financial transactions on a global scale and serve as a tool for financial inclusion of the poor.

\subsubsection{Financial Inclusion}

Cryptocurrencies and decentralized payment-systems could become an important tool for POs to further advance financial inclusion. They enable access to the financial system with almost no financial infrastructure requirements. A single post office would be able to provide various financial services with an internet connection being the only requirement. With this technology posts could provide a savings account, where money could be stored in cryptocurrencies. Individuals without a bank account but a postal outlet in near proximity might particularly benefit from this opportunity. In addition, it would be possible to include a service to use cryptocurrency for payments to other individuals or companies. For those without access to the financial system, POs could represent a reliable and trusted gateway to a payment system based on cryptocurrencies, which might look like a scam at first glance. Widespread use of cryptocurrencies for mobile phone payments reflects the fact that the general demand for additional payment methods is high in developing countries. Moreover, in a number of African countries such services have been introduced and are already used by more than 20 percent of all adults (see Lammer, 2014).

Exclusion from the financial system is an important issue and a major obstacle for participation in global commerce. According to the World Bank (see Lammer, 2014), over 2.5 billion adults in the world do not have a formal bank account. The percentage of socalled unbanked people is particularly high in developing countries, where also approximately 200 million smaller enterprises lack access to financial services and credits. Even developed countries, like the United States, are not immune from this issue. For those excluded from the financial system this means that they lack a secure way to save their money or to transfer it to other individuals. On a macro level, this also hinders economic 
participation and development, as a well-functioning financial system is one of the key enablers for growth.

POs could offer a partial solution to this problem. With their widespread physical presence, which also extends to rural and poor areas, they are well suited to provide a financial gateway for unbanked people incl. financial services. This especially applies to all areas with no bank in near proximity. In fact, about 20 percent of the people without an account state as a reason that banks are too far away to use (see Deminguc-Kunt and Klapper, 2012).

In this regard, the Universal Postal Union (2012) has set financial inclusion as an important objective for POs in the coming years. For POs there are different business models ranging from a pure cash merchant to a licensed financial service provider (see Universal Postal Union, 2013). There is some evidence that POs already contribute to financial inclusion to a certain extent: vulnerable groups, such as the poor, less educated, and those out of the labour force, are relatively more likely to use an account from POs than from other financial institutions (see Anson et al., 2013).

\subsubsection{International money transfers}

Besides offering a secure way to store wealth, cryptocurrencies also allow for efficient international money transaction. This is especially relevant for financial inclusion as remittances are important in developing countries. According to the World Bank (2014), migrants from developing countries have sent back USD 414 billion in earnings to their relatives in 2013. However, sending remittances through traditional channels is very costly, as such a service demands 9 percent of the transaction in fees on average.

With cryptocurrencies, it becomes possible to make international money transfers with only a minor fraction of the transaction fees of existing services. This would particularly help poor people to afford money transfers.

\subsubsection{Integration of financial and physical transactions}

Besides transmitting value, decentralized payment-systems are potentially capable of adding information and other functionalities to transactions. For instance, payment transaction data has a timestamp through inclusion in the blockchain. This timestamp could be used for reference in a parcel's track-and-trace information. Payment data may also contain shipping information, such that the PO and customs are automatically prenotified of goods to be expedited as soon as a payment hits the blockchain. In essence, decentralized payment-systems allow for a close link between the financial and the nonfinancial part of commercial transactions, which could unify payment and delivery in a single process.

Introduction of cryptocurrencies and decentralized payment-systems into postal business does not only enable new financial services by the posts, but it could also influence the logistic process and has the potential of innovating international parcels and mail delivery. Consequently, the Universal Postal Union considers decentralized paymentsystems as a potential way to simplify the complex system of international transactions, as they offer the possibility to synchronize financial and physical (logistics) transactions (see Anson, 2014). 


\section{Postcoin - A postal cryptocurrency}

A considerable drawback for cryptocurrencies is their high exchange rate volatility. Remedy could be provided by fully backing cryptocurrencies with other assets as well as by invoking a trusted party as issuer. Such improvements of cryptocurrencies give rise to the idea of a Postcoin. Postcoins could be issued by a PO by tagging (or "coloring" $)^{13}$ existing digital tokens on a public blockchain, e.g. Bitcoins, to represent a specific asset. The post would sell each unit of Postcoin for a certain amount of local currency, while holding the equivalent value of a defined unit of this asset (e.g. USD, gold or SDR) as reserve. ${ }^{14}$ At the same time it would also guarantee to buy back every Postcoin for local currency at an amount at least equivalent to its value in terms of reserves. Thereby, Postcoins could be injected into the economy via an exchange between the respective PO and its customers. Issuing a Postcoin, posts could make use of the open infrastructure of public blockchains and at the same time be in control of the money supply as well as the access and use of their permissive Postcoin currency. The reputation of POs constitutes a key factor in this regard.

\subsection{Advancing cryptocurrencies}

Compared to other cryptocurrencies, Postcoin would exhibit the advantage that it does not suffer from volatility: it would offer a stable store of value by virtue of not only being issued by a reliable source but also being pegged to another asset or currency, while still being fast and cheaply transferable on a public blockchain. In essence, Postcoin would enable customers to benefit from all the advantages of cryptocurrencies, while adding a trusted institution to interact with.

Providing a postal currency could solve another issue related to cryptocurrencies: it may be somewhat difficult to handle the plethora of unsystematic information on cryptocurrencies as well as to fully understand their concept. Where the public largely views a PO as a trusted authority, Postcoin could become an alternative cryptocurrency which is both easy to understand and to use. All necessary information can be provided by the post as a reliable institution. The post could offer Postcoin account services at postal franchises and integrate Postcoin accounts into postal websites. Consequently, customers would need no advanced technical knowledge on cryptocurrencies, but would still be able to use the corresponding services such as payments and money transfers.

\subsection{Further potentials of Postcoin}

The concept of Postcoin could also serve as a successful business model for countries with unstable national currencies. If people trust the postal system more than the central

${ }^{13}$ Colored coins are a method for associating assets with tokens on a blockchain network.

${ }^{14}$ If a PO issues its own currency, it has full control over its supply and essentially takes the place of a central bank. By coloring digital tokens, it puts a layer on another cryptocurrency and uses it as a payment system. All transactions in Postcoins are at the same time transactions in the other cryptocurrency which are verified in the other cryptocurrency's network (and which have to pay according transaction fees). 
money issuer of their country, Postcoin might emerge as an important store of wealth relative to the national currencies. This could also assist in stabilizing the economy of the respective country. Moreover, it is conceivable that each national PO would create its own Postcoin with international payments being settled between operators. The Universal Postal Union might then act as an exchange between different postal currencies.

It would be even more efficient if the international postal community (national incumbents and competitors ${ }^{15}$ ) issued one single postal currency with the Universal Postal Union coordinating the efforts of national POs. Such coordination measures might entail regulations, standards and multilateral agreements (see Anson, 2014). In particular, the Universal Postal Union would need to ensure that all POs issuing Postcoin adhere to the same exchange rates and reserve standards in an auditable way. If all POs were to coordinate on a single Postcoin currency, the reputation effects would amplify due to the participation of many trusted parties.

Besides, Postcoin services could be implemented at a smaller scale and act as a bonus feature rather than a core part in the business model. Comparable to frequent flyer miles, Postcoin could reward loyal customers and give rights to additional benefits (e.g. exchange for postal services). A bonus system might also be a safe way to test Postcoin as a payment system before launching it as a full-fledged cryptocurrency.

\section{Other commercial applications of blockchain technology}

Blockchain technology offers business opportunities for POs beyond currency and financial services, e.g. in identity services, device management or supply chain management (see USPS, 2016).

Identity Services POs could offer identity verification services, both for persons and goods. A verified personal digital identity would allow users to know that the peers they are transacting with are real and have proof of ownership. POs could further link that virtual identity used by the customer to operate within a blockchain system with real-world identifiers, such as a person's postal address. Customers could use these verified identities to login to secure websites, notarize documents, or participate in smart contracts. Likewise, identification of goods would allow linking them to digital tokens and track their ownership on a blockchain.

Device Management Another potential application of blockchain technology would be using it to secure and maintain the Internet of (postal) Things. The blockchain's decentralized control and verification system could allow devices to more securely record and transfer data. This would also help increase the security of the network by removing the risks associated with single points of access. With blockchain technology, networks of devices would be able to transact with other connected devices to, for example, pay for services, share power resources or contract for maintenance services and part replacement.

Supply Chain Management A final application would be better supply chain management: using blockchain to identify packages and mail. POs have a number of customers,

${ }^{15}$ Provided that they comply with the required regulations. 
partners, contractors and other stakeholders that it coordinates with. Tracking mailpieces on a blockchain would allow POs to keep an auditable chain of custody and embed additional shipment and tracking information to facilitate customs clearance and faster delivery. If a mailpiece was embedded with a sensor, it could keep track of its own chain of custody while executing smart contracts for payment and customs clearance. Generally, blockchain technology would allow for close linkages between the financial, logistics, and delivery parts of commercial transactions with the power to unify payment and delivery in one seamless experience. ${ }^{16}$ POs could become a single intermediary between merchants and customers, allowing them to reduce coordination needs, offer more efficient ecommerce solutions, contribute to the growth of ecommerce (particularly cross-border ecommerce), and increase their market share and revenue.

\section{Conclusion}

In the past few years, the publicity for blockchain technology and cryptocurrencies has increased. They enable transfer of value across the Internet just as emails transfer information. In particular, this means that people can make peer-to-peer transactions without financial intermediaries in between. Furthermore, blockchain-based payment-systems are completely decentralized and lack central institutions. Instead, they are controlled by the community of their users. With their unique characteristics such as low transaction costs and secure transactions, decentralized payment-systems can offer various benefits for individuals, companies and the society as a whole. Nevertheless, there are still some challenges. Most importantly, the exchange rate of cryptocurrencies is highly volatile and decentralized payment-systems lack any established institution for people to rely on.

POs are well-suited to counter some of the main weaknesses. They combine a widespread physical network with a strong reputation as well as with substantial experience in financial service provision. Financial services such as savings accounts or money transfers could rather easily be extended internationally and thus assist in advancing financial inclusion. Posts could also issue their own postal cryptocurrency, the Postcoin, which could be capable of protecting customers from the high exchange-rate volatility that cryptocurrencies currently bear.

Looking into the future, the innovative technology of decentralized payment-systems is likely to persist and to transform the existing financial system. Institutions like posts as well as regulators should therefore keep up with any future developments.

\section{References}

Anson, José. (2014). Supporting Transactions not Pegged to National Currencies: A Role for the UPU. USPS OIG-UPU International Online Forum on Postal Innovation 2014.

Anson, José; Berthaud, Alexandre; Klapper, Leora and Singer, Dorothe (2013). Financial Inclusion and the Role of the Post Office. World Bank Policy Research Working Paper 6025 .

${ }^{16}$ See Section 3.2.3. 
Bloomberg (2014a). Bitcoin Prices Plunge on Report PBOC Orders Accounts Shut. Available at http://www.bloomberg.com/news/2014-03-27/pboc-orders-banks-to-shutbitcoin-exchange-accounts-caixin-says.html.

Bloomberg (2014b). Mt. Gox Bitcoin Exchange Down amid \$365 Million Theft Claim. Available at http://www.bloomberg.com/news/2014-02-25/mt-gox-bitcoin-exchangegoes-offline-as-peers-lash-out-at-firm.html.

Coindesk (2014). Bitcoin vs Argentine Peso: Which is worse off? Available at http://www.coindesk.com/bitcoin-vs-argentine-peso-worse/.

EMarketer (2013). B2C Ecommerce Climbs Worldwide, as Emerging Markets Drive Sales Higher. Available at http://www.emarketer.com/Article/B2C-Ecommerce-ClimbsWorldwide-Emerging-Markets-Drive-Sales-Higher/1010004.

Financial Crimes Enforcement Network (2013). Application of FinCEN's Regulations to Persons Administering, Exchanging, or Using Virtual Currencies. Available at http://www.fincen.gov/statutes_regs/guidance/pdf/FIN-2013-G001.pdf.

Financial Crimes Enforcement Network (2014a). Application of FinCEN's Regulations to Virtual Currency Mining Operations. Available at http://www.fincen.gov/news_room/rp/rulings/pdf/FIN-2014-R001.pdf.

Financial Crimes Enforcement Network (2014b). Application of FinCEN's Regulations to Virtual Currency Software Development and Certain Investment Activity. Available at http://www.fincen.gov/news_room/rp/rulings/pdf/FIN-2014-R002.pdf.

Jaag, Christian and Christian Bach (2013). Virtual Currencies and Physical Posts: A Perfect Match. The Postal Industry, 1(2), 11-12.

Jaag, Christian and Christian Bach (2015). The Effect of Payment Reversibility on ECommerce and Postal Quality. Handbook of Digital Currency, Chapter 6, Elsevier.

Jaag, Christian and Andreas Haller (2016). Economic mechanism design in Bitcoin: Mining, block formation and transaction fees. Swiss Economics Working Paper 57.

Lammer, Thomas (2014). Virtual currencies, Payment Systems \& Financial Inclusion. USPS OIG-UPU International Online Forum on Postal Innovation 2014.

Nasdaq (2016). The Halving: Then \& Now - a Bitcoin Magazine Infographic. Available at http://www.nasdaq.com/article/the-halving-then-now-a-bitcoin-magazine-infographiccm646839\#ixzz4EDstlcdw

New York State Department of Financial Services, NYDFS (2015). Press Release NYDFS Announces Approval of first BitLicense application from a virtual currency firm. September 22.

The Economist (2015). The Trust Machine. October 31.

The Street (2014). Three in Four People Have Never Heard of Bitcoin. Available at http://www.thestreet.com/story/12306983/1/three-in-four-people-have-never-heard-ofbitcoin.html.

United States Postal Service, USPS (2016). Blockchain Technology: Possibilities for the U.S. Postal Service, RARC Report RARC-WP-16-011. 
Universal Postal Union (2014). Development of Postal Services in 2013. Available at http://www.upu.int/fileadmin/documentsFiles/resources/postalStatistics/developmentO fPostalServicesIn2013En.pdf.

Universal Postal Union (2013). Global Panorama on Postal Financial Inclusion: Business Models and Key Issues. Available at www.upu.int/fileadmin/documentsFiles/activities/financialInclusion/publicationGlobal PanoramaFinancialInclusionEn.pdf.

Universal Postal Union (2012). Annual Report 2012. Available at http://news.upu.int/insight/annual-reports/2012/.

Watkins, Kevin and Maria Quattri (2014). Lost in intermediation - How excessive charges undermine the benefits of remittances for Africa. Overseas Development Institute Report.

World Bank (2014). Migrants from Developing Countries to Send Home \$414 Billion in Earnings in 2013. Available at

http://www.worldbank.org/en/news/feature/2013/10/02/Migrants-from-developingcountries-to-send-home-414-billion-in-earnings-in-2013. 